\title{
EDUCACIÓN MÉDICA CONTINUA
}

\section{SÍNDROME HEPATOPULMONAR}

\author{
Álvarez Arguedas, Karina \\ Médico General, Servicio de Urgencias del Hospital Monseñor Sanabria, Puntarenas, Costa Rica.
}

\begin{abstract}
Resumen: El Síndrome Hepatopulmonar (SHP) es un síndrome subdiagnosticado que se define como una tríada clínica de enfermedad hepática crónica, desoxigenación y dilatación vascular pulmonar. Es una complicación rara de la enfermedad hepática que indica mal pronóstico. Muchas teorías se han propuesto para aclarar su fisiopatología. Las mayores manifestaciones clínicas son hipoxemia arterial, hipocratismo digital, telangectasias, ortodeoxia y platipnea. No se ha encontrado un tratamiento médico efectivo, aunque el trasplante hepático se ha considerado capaz de revertir, al menos parcialmente esta situación. Terapia con oxígeno a largo plazo pre y postrasplante puede ofrecer una nueva opción terapéutica para mejorar la función hepática en pacientes con cirrosis e hipoxemia.
\end{abstract}

Palabras clave: Síndrome hepatopulmonar, Desoxigenación arterial, Dilatación vascular pulmonar, Ecocardiografía de contraste

Abstract: Hepatopulmonary syndrome (HPS) is an underdiagnosed syndrome that is defined as a clinical triad of advanced liver disease, arterial deoxygenation and intrapulmonary vascular dilatation. It is a rare complication of liver disease of varied etiologies and indicates a poor prognosis. Many theories have been put forward to throw light over its pathogenesis. The major clinical manifestations are arterial hypoxemia, clubbed fingers and spider naevi. Orthodeoxia and platypnea are usual clinical features. A simple non invasive method to screen HPS is desirable. Contrast enhanced 2D Echo cardiography is the preferred screening test. No effective medical treatment has been found. Although liver transplant seems feasible to reverse, at least partially, this situation. Long-term oxygen therapy pre and post liver transplant might offer a new therapeutic approach to improve liver function in patients with cirrhosis with hypoxemia.

Key words: Hepatomulmonary syndrome, Arterial deoxigenation, Intrapulmonar vascular dilatation, Contrast echo cardiography, Liver transplant

Recibido: 19 marzo 2012. Aceptado: 10 abril 2012. Publicado: 27 abril 2012. 


\section{INTRODUCCIÓN}

La relación entre la enfermedad hepática y pulmonar fue descrita por primera vez por Fluckiger en 1884 basado en la observación de una mujer con cirrosis, cianosis y acropaquias [1]. El término SHP fue propuesto por primera vez por Kennedy y Knudson para describir la cianosis que se desarrolló en un paciente con cirrosis hepática cuatro años después de una cirugía de un circuito porto-caval [2].

La asociación de la disfunción hepática con hipoxemia en ausencia de enfermedad cardiopulmonar intrínseca es conocida como SHP [3].

El SHP es caracterizado por un defecto en la oxigenación arterial inducido por vasodilatación vascular pulmonar en enfermedad hepática establecida [4]. Este síndrome clínico consta de tres componentes: enfermedad hepática, dilatación vascular pulmonar y un defecto en la oxigenación [4].

El componente vascular incluye dilatación de los capilares pulmonares locales y difusos y menos comúnmente comunicaciones arteriovenosas pleurales y pulmonares [4].

La hipoxemia arterial es común en el contexto de enfermedad hepática, su causa es frecuentemente multifactorial (ascitis, hidrotórax hepático y enfermedad pulmonar obstructiva crónica) [4]. La definición de hipoxemia arterial asociada a SHP está basada en las medidas de la presión parcial de oxígeno que se presentan en un paciente en una posición estandarizada, preferiblemente sentado o decúbito supino[4]. El uso del gradiente de oxígeno alveolocapilar es importante debido a que puede aumentar anormalmente antes de que la presión parcial arterial de oxígeno por sí misma se torne anormalmente baja. Esto ocurre en respuesta a la medida en que el gradiente compensa los niveles disminuidos de dióxido de carbono arterial, además de la hiperventilación que se compensa con alcalosis respiratoria, situación que es común en los pacientes con cirrosis [4].

Las siguientes patologías son las que más se relacionan con el riesgo de desarrollar SHP:

Tabla \#1. Riesgo de desarrollar SHP según patología

\begin{tabular}{|l|l|}
\hline Patología & Riesgo \\
\hline Cirrosis & $5-30 \%$ \\
\hline Hipertensión Portal no Cirrótica & $4-8 \%$ \\
\hline
\end{tabular}
$[5,6]$
Pacientes con cirrosis o hipertensión portal que esperan trasplante pueden desarrollar SHP hasta en un $25 \%$ de los casos [7].

Es importante buscar comunicaciones izquierda-derecha de cámaras cardiacas en pacientes que tienen enfermedad pulmonar con hipoxemia marcada y de esta foma lograr descartar causas de origen cardiaco [8].

El SHP y la Hipertensión Portopulmonar (HPP), deben ser estrictamente diferenciadas ya que tienen origen histológico y fisiopatológico diferente [9]. Mientras que el SHP es una enfermedad dilatadora de la vasculatura pulmonar, la HPP es una enfermedad constrictora y obliterativa de la vasculatura pulmonar en el contexto de enfermedad hepática o de hipertensión portal, por lo tanto son entidades separadas tanto para diagnóstico como para tratamiento [9].

El SHP se puede clasificar de acuerdo a los dos tipos de dilataciones vasculares que se pueden presentar basados en la angiografía pulmonar $[1,10]$.

\section{ETIOLOGÍA}

La etiología de este síndrome no está claramente establecida [1], pero entre los factores contribuyentes se destacan las causas más comunes de cirrosis que son hepatitis $\mathrm{C}$ y alcoholismo en un $55 \%$ de los casos [11].

\section{FISIOPATOLOGÍA}

La patogénesis no está bien establecida pero existe una asociación de factores como un desbalance en la respuesta del receptor de endotelina, remodelamiento microvascular pulmonar y predisposición genética [12].

La patología principal en la que se basa el desarrollo del SHP es la dilatación capilar y precapilar de la vasculatura intrapulmonar. También se han determinado comunicaciones arteriovenosas pleurales y pulmonares y anastomosis portopulmonares [13].

La sangre venosa mixta pasa a través de los capilares pulmonares por los circuitos intrapulmonares de una forma muy rápida y muy lejos de la membrana alveolar por lo que no logran un adecuado intercambio gaseoso, causando un desequilibrio ventilaciónperfusión y consecuentemente hipoxemia.

Revista electrónica publicada por el Departamento de Farmacología de la Escuela de Medicina de la Universidad de Costa Rica, 2060 San José, Costa Rica. ${ }^{\circledR}$ All rights reserved. Licensed under a Creative Commons Unported License. 
Tabla \#2. Clasificación del SHP de acuerdo a sus lesiones vasculares.

\begin{tabular}{|l|c|c|}
\hline & Lesiones Tipo I & Lesiones Tipo II \\
\hline Frecuencia & Alta & Baja \\
\hline Respuesta $\mathrm{a} \mathrm{O}_{2}$ & Buena & Pobre \\
\hline Patrón & Difuso & Localizado \\
\hline Tratamiento & Trasplante hepático & Embolización \\
\hline
\end{tabular}

$[1,10]$

Esta alteración genera un tono vascular pulmonar relativamente compensado, el cual es menos capaz de acomodar el cambio del flujo sanguíneo gravitacional en unidades alveolares dependientes y resulta en ortodeoxia [14]. La dilatación vascular también puede exacerbarse por la circulación hiperdinámica que es común en pacientes cirróticos [15].

En el SHP avanzado ocurre un desmejoramiento de la difusión alveolocapilar, probablemente por la interface alveolocapilar que la complica. El aumento de la producción pulmonar de óxido nítrico, un potente vasodilatador, ha sido implicado como un protagonista en el SHP. Esto se ha determinado por el aumento de los niveles de óxido nítrico exhalado en SHP y su normalización seguida del trasplante hepático [13]. La fisiopatología detrás de esta entidad es compleja e involucra además, diferentes sustancias vasoactivas que están relacionadas con el óxido nítrico como lo es la endotelina-1[16].

El mecanismo exacto no está claro pero en estudios realizados en ratas con ligadura del ducto biliar, se determinó que la endotelina-1 producida por el hígado, posiblemente actúa por la vía de los receptores de endotelina-b para estimular la óxido nítrico sintasa en el endotelio de la vasculatura pulmonar y de esta forma estimula la vasodilatación $[17,18]$.

La acumulación de macrófagos intravasculares a nivel pulmonar probablemente incrementados por la endotoxinemia causada por la traslocación intestinal de bacterias en la enfermedad hepática, estimulan la expresión y la actividad de la óxido nítrico sintasa y de la hemo oxigenasa -1, induciendo la sobreproducción de óxido nítrico y de monóxido de carbono, resultando en vasodilatación de la vasculatura pulmonar $[17,18]$.

También se ha determinado que la Norfloxacina disminuye la acumulación de macrófagos y normaliza la actividad de inducción de la óxido nítrico sintasa en modelos animales, sugiriendo que la endotoxinemia que se produce en la enfermedad hepática puede estimular la acumulación de macrófagos y contribuir al desarrollo del SHP [19].

Además, se han realizado estudios que han logrado identificar más específicamente la acumulación de macrófagos CD68 en los casos de SHP [20].

Estudios recientes han reportado que la angiogénesis también es un factor importante en la patogénesis del SHP y que la inhibición de ésta puede ser un potencial tratamiento en el futuro [21].

Se han documentado polimorfismos en genes involucrados en la regulación de la angiogénesis, generando un riesgo intrínseco para desarrollar SHP. Cuarenta y dos polimorfismos de un nucleótido simple en 21 genes se asociaron fuertemente el SHP después de ajuste para raza y fumado. De estos, 8 presentaron al menos 2 polimorfismos de un nucleótido simple asociados a la enfermedad: CAV3, ENG, NOX4, ESR2, VWF, RUNX1, COL18A1, y TIE1 [22].

\section{MANIFESTACIONES CLÍNICAS}

Los signos que sugieren SHP incluyen telangectasias, hipocratismo digital y cianosis. Aproximadamente el $18 \%$ de los pacientes con SHP presentan disnea. La dificultad para respirar aparece en bipedestación (platipnea) y se acompaña de desaturación de oxígeno en esa posición (ortodeoxia) [15]. La Ortodeoxia-platipnea se define como un descenso de la presión parcial de oxígeno de al menos un $5 \%$, o de $4 \mathrm{mmHg}$ en bipedestación con respecto al decúbito supino y es altamente específico para SHP [15].

La prevalencia de estos síntomas se estima en $5 \%$ de pacientes portadores de cirrosis y en $88 \%$ de pacientes con SHP [23].

El hipocratismo digital presenta el valor predictivo positivo más alto para el diagnóstico (75\%) y la disnea presenta el mayor valor predictivo negativo 


\section{(100\%) [24].}

Para el gradiente alveolocapilar los valores predictivos positivos fueron $37 \%$ y $53 \%$ y para la presión arterial de oxígeno (PaO2) fueron de 93-94\% [24].

En las pruebas de función pulmonar el hallazgo más común encontrado es un descenso en la capacidad de difusión del monóxido de carbono [25].

\section{DIAGNÓSTICO}

En la evaluación del paciente cirrótico con hipoxemia es indispensable realizar una detallada historia clínica y un buen examen físico que ayudará a determinar diagnósticos alternativos de disnea. Es necesario excluir otras causas cardiopulmonares contribuyentes como atelectasias pulmonares, ascitis, enfermedad pulmonar obstructiva crónica (EPOC) o hidrotórax hepático [26].

El diagnóstico definitivo de SHP requiere ecocardiografía de contraste, centellografía con Tecnesio 99 con albumina macroagregada, o angiografía pulmonar [23].

El ecocardiograma de contraste transtorácico o transesofágico es el método diagnóstico más recomendado para la detección de la dilatación vascular intrapulmonar [15]. Es más sensible y menos invasivo que la angiografía [23]. Éste es capaz de determinar circuitos intrapulmonares y los distingue de los circuitos intracardiacos [27].

Aunque el circuito arteriovenoso es diagnosticado como positivo en el ecocardiograma de contraste, ocurre sólo en un $13-47 \%$ de pacientes con enfermedad hepática crónica, y la incidencia de hipoxemia en estos pacientes ronda apenas con cifras de 5 a $13 \%$ [28].

La centellografía con Tecnesio 99-m con albúmina macroagregada es un procedimiento diagnóstico alternativo [15]. Este revela un aumento de la actividad del radionucleótido en el cerebro y en el riñón, porque la mayoría de albúmina macroagregada que queda normalmente atrapada en la circulación pulmonar pasa a través de los vasos dilatados y alcanzan a estos órganos. Sin embargo esto también ocurre en circuitos intracardiacos [1]. Este método confirma el circuito arteriovenoso, pero no puede ser utilizado para determinar el nivel del circuito [27].

La angiografía pulmonar es el método invasivo estándar y es capaz de diferenciar entre lesiones tipo I y tipo II[1]. Se considera este método si la presión parcial de oxígeno en sangre con inspiración del 100\% de $\mathrm{O}_{2}$ es menor de $150 \mathrm{mmHg}$ y su objetivo es detectar comunicaciones arteriovenosas que se puedan tratar con embolización en el caso de las lesiones tipo II[15].

El gradiente de oxígeno alveolocapilar es el más valioso predictor diagnóstico positivo o negativo para la presencia de SHP en pacientes cirróticos [29].

Este gradiente es un parámetro más sensible que la $\mathrm{PaO} 2$ cuando se valoran las alteraciones en el intercambio gaseoso. Un gradiente alveolocapilar mayor a $15 \mathrm{mmHg}$ o mayor a $20 \mathrm{mmHg}$ en pacientes mayores de 64 años respirando aire ambiente ha sido ampliamente recomendado como criterio diagnóstico para SHP [15].

Una clasificación de la severidad del síndrome hepatopulmonar basado en anormalidades de oxigenación es fundamental porque la severidad va a influir en la sobrevivencia y es útil en determinar el tiempo y los riesgos de trasplante hepático [4].

La severidad del SHP está basada en los valores del gradiente de la presión alveolo-arterial de oxígeno [P(A-a) 02] e incluye cuatro estadíos:

Tabla \#3. Severidad del SHP basada en gradiente de la presión alveolocapilar y en la presión arterial de oxígeno.

\begin{tabular}{|c|c|}
\hline LEVE & $\mathrm{P}(\mathrm{A}-\mathrm{a}) \mathrm{O}_{2} \geq 15$ y $\mathrm{PaO}_{2} \geq 80$ \\
\hline MODERADO & $\mathrm{P}(\mathrm{A}-\mathrm{a}) \mathrm{O}_{2} \geq 15$ y $\mathrm{PaO}_{2} \geq 60$ y $<80$ \\
\hline SEVERO & $\mathrm{P}(\mathrm{A}-\mathrm{a}) \mathrm{O}_{2} \geq 15$ y $\mathrm{PaO}_{2} \geq 50$ y $<60$ \\
\hline $\begin{array}{l}\text { MUY SEVERO } \\
\mathrm{O}_{2} \mathrm{~S}\end{array}$ & $\begin{array}{l}\mathrm{P}(\mathrm{A}-\mathrm{a}) \mathrm{O}_{2} \geq 15 \text { y } \mathrm{PaO}_{2}<50 \\
\text { (o }<300 \mathrm{mmHg} \text { con inspiración de } 100 \%\end{array}$ \\
\hline
\end{tabular}

[15]. Valores están dados en mmHg.

\section{TRATAMIENTO}

En la actualidad no se cuenta con terapia médica disponible. Los resultados de tratamientos médicos (inhibidores de vasodilatación como almitrina, indometacina, ocreótido, antibióticos) han sido insatisfactorios y fueron evaluados solo en pequeños grupos de pacientes [15].

Se han realizado estudios para determinar el papel de la antibioticoterapia con norfloxacina, ésta ha logrado ejercer un efecto en la inhibición de la dilatación vascular pulmonar causada por la endotoxinemia, pero no se determinó que causara 
mayor efecto en el intercambio gaseoso en pacientes con SHP [30].

Estudios en animales han demostrado que el SHP puede ser prevenido con pentoxilina [31]. Éste es un inhibidor de factor de necrosis tumoral que actúa probablemente disminuyendo la angiogénesis, inhibe directamente la activación de óxido nítrico y bloquea la acumulación de monocitos a nivel intravascular, por lo que mejora el SHP a nivel experimental [32, 33].

En otros estudios de pacientes con SHP se determinó que la pentoxilina no mejoraba la oxigenación arterial y que su tolerancia fue muy limitada por efectos gastrointestinales [34].

En una publicación reciente se destacó la importancia de la suplementación dietética con ácido gárlico en pacientes con SHP. Se ha reportado que éste ha logrado revertir las comunicaciones intrapulmonares, mejora la hipoxemia y disminuye la mortalidad inhibiendo la producción de óxido nítrico por los macrófagos, pero todavía es necesario realizar más estudios [35].

Algunas técnicas intervencionistas como embolización vascular de comunicaciones arteriovenosas [4, 15], que es una opción para pacientes SHP con lesiones tipo II, o descompresión portal con circuito intrahepático portosistémico transyugular, pueden ayudar a mejorar la $\mathrm{PaO} 2$ reduciendo la hipertensión portal[1]. Sin embargo los pocos datos disponibles, y el riesgo de exacerbar un estado circulatorio hiperdinámico, que a su vez puede inducir vasodilatación intrapulmonar y aumentar la severidad del SHP no brinda el soporte necesario para su uso como terapia paliativa [4].

La cavoplastía ha demostrado ser una terapia efectiva para el SHP cuando está asociado al HPP [7].

Paracentesis y oxigenoterapia han provisto de alivio sintomático [4, 36].

El mejoramiento espontáneo del SHP fue descrito en algunos casos cuando la enfermedad hepática fue resuelta o cuando se desarrolló hipertensión pulmonar [15].

Se han reportado casos de SHP que ocurren en pacientes no cirróticos con hipertensión portal que revierten totalmente después que el agente causal es erradicado [1].

\section{Trasplante hepático}

A pesar de la relación que existe entre el trasplante hepático y el aumento en la mortalidad, es el único tratamiento disponible efectivo para el SHP establecido [37].

Éste brinda mejoría en la resolución de la hipoxemia en un $80 \%$ de los pacientes, aunque requiera algunos meses después del trasplante (desde 3 meses hasta 1 año) [38]. Cada posible candidato para trasplante hepático debe ser evaluado y estudiado por enfermedades extrahepáticas una vez que este síndrome es confirmado. La severidad y progresión del SHP a través del tiempo deben ser supervisadas para optimizar el tiempo de trasplante en los pacientes que cumplan con los criterios de excepción de MELD[38].

El puntaje MELD (Modelo de Enfermedad Hepática en Fase Terminal) es un predictor de sobrevivencia y se utiliza para priorizar las listas de espera de pacientes con enfermedad hepática crónica para trasplante hepático, está basado en simples valores de laboratorio rutinarios (bilirrubina, INR y creatinina)[39]. Aunque este sistema de puntaje fue introducido desde el año 2002, no ha sido utilizado en muchos de los estudios de trasplante hepático con SHP [40].

Además, existe la política 3.6.4.5.1 de la Red Unida para Trasplante de Órganos (UNOS) que otorga puntos de excepción a pacientes con el diagnóstico de SHP para determinar su riesgo de mortalidad [39].

En estudios recientes se plantean importantes preguntas acerca de la exactitud del diagnóstico de SHP en pacientes que reciben puntos de excepción con los sistemas anteriormente mencionados y la necesidad de criterios más estandarizados de la gravedad y la progresión de SHP [41].

Posterior al trasplante hepático el $24 \%$ de los pacientes requieren oxígeno al $100 \%$ por $11-31$ días postrasplante y los periodos de hospitalización en algunos casos han superado los 3 meses [42].

Se ha planteado la cuestión de si los buenos resultados postrasplante son por trasplante temprano, o por mejoría en el manejo[41].

Un mejor rastreo de información de pacientes con SHP podría generar una igualdad en la asignación para trasplante y mejorar la definición de la historia natural del SHP [41].

Complicaciones biliares y vasculares fueron las más frecuentes que se han reportado en la rutina de trasplante hepático. Éstas son agravadas por hipoxia tisular. En futuros manejos se debe procurar lograr mayores saturaciones de oxígeno en periodos peri y post operatorios [40, 42].

Existen estudios en los que se ha determinado que la embolización pulmonar postrasplante puede ser un tratamiento útil para acelerar la recuperación y disminuir el riesgo de morbilidad y mortalidad en pacientes en los que falla la recuperación temprana postrasplante. Más estudios se necesitan para

Revista electrónica publicada por el Departamento de Farmacología de la Escuela de Medicina de la Universidad de Costa Rica, 2060 San José, Costa Rica. ${ }^{\circledR}$ All rights reserved. Licensed under a Creative Commons Unported License. 
confirmar estos hallazgos [43].

\section{Oxígeno suplementario}

La oxigenoterapia a largo plazo ha sido de las terapias más frecuentemente recomendadas para pacientes portadores de SHP con hipoxemia severa, aunque la confianza de este tratamiento, su eficacia y su valor costo beneficio no ha sido establecido [4].

Los pacientes con SHP tienen una disminución importante de la saturación de 02 a través del tiempo comparados con pacientes sin SHP [44]. Se ha determinado que la hipoxemia es un predictor independiente de la sobrevivencia del paciente portador de SHP y además puede contribuir con el deterioro de la función hepática [45].

En un estudio se les administró oxígeno suplementario en bajas dosis a pacientes con SHP para mejorar los síntomas respiratorios, sorprendentemente la función hepática mejoró de Child Pugh clase $\mathrm{C}$ a clase A, y la ascitis desapareció después de un año de administrar oxígeno suplementario a estos pacientes [45]. Se manifiesta que la terapia con oxígeno a largo plazo ha contribuido en la mejoría de la función hepática en los casos estudiados [45].

En un estudio de trasplante hepático se determinó que la aplicación de ventilación no invasiva aplicada inmediatamente después del trasplante hepático ha sido efectiva para la prevención de complicaciones postoperatorias severas como reintubación y la muerte hospitalaria [46].

\section{PRONÓSTICO}

El curso del SHP es progresivo y está asociado a una alta morbilidad y mortalidad [47].

El pronóstico de pacientes con SHP que no se someten a trasplante hepático es pobre. Un estudio clínico prospectivo de pacientes con cirrosis hepática reveló que pacientes con SHP a los que no se les realizó trasplante presentaron 3,8 veces menor sobrevivencia de la media comparada con los pacientes que no portaban SHP. Se ha determinado una sobrevivencia media de 10,6 meses si no se realiza trasplante hepático en pacientes con SHP [36].

Sin embargo una mortalidad considerable fue asociada al trasplante hepático en SHP [36]. En estudios recientes se ha indicado una mortalidad postrasplante con tasas de $16 \%$ con SHP, otros estudios han reportado cifras de hasta un $30 \%$ de mortalidad postrasplante [36].

Algunos estudios recientes mencionan una sobrevivencia de hasta $76 \%$ a 5 años, un rango no muy diferente a pacientes sin SHP [7,23].

El mayor predictor de muerte fue una presión parcial de 02 preoperatoria menor de $50 \mathrm{mmHg}$ [4]. Las causas de muerte raramente son atribuidas a disfunción pulmonar. La mortalidad está mayormente asociada a sangrado digestivo alto y a disfunción hepática [15].

Desde que el SHP es un factor independiente del pronóstico de pacientes con cirrosis, los pacientes con este diagnóstico deberían ser prioritarios en las listas de espera de trasplante [15].

\section{CONCLUSIONES}

El SHP es una complicación de la enfermedad hepática crónica que es subdiagnosticada.

Se requieren más estudios para aclarar su etiología y patogénesis.

Este síndrome puede ser tratado con oxígeno, pero se puede corregir solo resolviendo la enfermedad de fondo o con trasplante hepático.

El SHP debe ser considerado en todos los pacientes con enfermedad hepática crónica que cursen con disnea e hipoxemia.

\section{RECOMENDACIONES}

Es necesario realizar estudios en nuestro país para lograr detectar la mayoría de casos de pacientes portadores de SHP, determinar su incidencia y explorar opciones terapéuticas viables para poder beneficiar a pacientes con esta patología.

\section{REFERENCIAS}

1. Agrawal G, N Kumar, D Rosha. Hepatopulmonary Syndrome. Japi, April 2008; vol (56):265-67.

2. Naeije Robert Hepatopulmonary syndrome and portopulmonary hypertension. Swiss Med Wkly 20 03, (1 33 3): $163-169$.

3. Young Hun Choi, Whal Lee, Jung-Eun Cheon, Woo Sun Kim, In-One Kim, Jae Hyung Park, Kyung Mo Yeon. CT findings in unilateral hepatopulmonary syndrome after the Fontan operation Pediatr Radiol 2009; (39):336-342. 
4. Rodríguez Roisin Roberto . MD, Michael J. Krowka. Hepatopulmonary Syndrome - A Liver induced Lung Vascular Disease MD: New England Journal of Medicine 2008; (358): 2387.

5. Kochar R; Nevah Rubin MI; Fallon MB Pulmonary complications of cirrhosis. Current Gastroenterology Reports 2011 Feb; 13 (1): pp. 34-9.

6. Maganty K; Ghanta R; Bejarano P; Weppler D; Tekin A; Moon J; Nishida S; Tzakis A; et al. Transplantation for hepatopulmonary syndrome due to noncirrhotic portal h ypertension. Transplantation Proceedings. 2011 Sep; 43 (7): pp. 2814-6.

7. Valenti A; Caimi G. Physiopathological, clinical and therapeutic aspects of hepatopulmonary syndrome. La Clinica Terapeutica 2010; 161 (3):123-8.

8. Tercé G; Stervinou-Wemeau L; Leroy S; HachullaLemaire; Wallaert B. Hepatopulmonary syndrome and diffuse interstitial lung disease. Revue Des Maladies Respiratoires. 2010 Nov; 27 (9):1114-8.

9. Kerstin Herzer, Felix Post, Ali Canbay, Guido Gerken. Hepatopulmonales Syndrom und portopulmonale Hypertonie Med Klin 2010; (105):916-23.

10. Khalid F. Almoosa, MD. The Hepatopulmonary Syndrome. Hospital Physician. June 2000; (2) 3 - 30.

11. Hamamoto J; Toba S; Hirosako S; Nakamura K; Fujii $\mathrm{K}$; Kohrogi H. A case of hepatopulmonary syndrome with elevated exhaled nitric oxide. The Journal of The Japanese Respiratory Society 2010 May; 48 (5): 37984.

12. Gonçalves de IVlacêdo Liana, Edmundo Pessoa de Almeida Lopes. Hepatopulmonary syndrome: an update Sao Paulo Med J. 2009; 127(4):223-30.

13. Symposium on Liver Disease. Pulmonary complications in liver disease. British Journal of Hospital Medicine. 2009 December; 70(12) 685-90.

14. Gomez FP, Martinez-Palli G, Barbera JA, Roca J, Navasa $M$, Rodriguez-Roisin $R$ Gas exchange mechanism of orthodeoxia in hepatopulmonary syndrome. Hepatology. 2004; (40): 660-6.

15. Krenke Rafał , E. Magdalena Grabczak, Urszula Ołdakowska-Jedynak, Ryszarda Chazan, Marek Krawczyk Hepatopulmonary síndrome
Experimental and Clinical Hepatology. 2010; 6(2): 27-29.

16. Pham DM; Subramanian R; Parekh S Coexisting hepatopulmonary syndrome and portopulmonary hypertension: implications for liver transplantation. Journal of Clinical Gastroenterology 2010 Aug; Vol. 44 (7): 136-40.

17. Zhang J, Ling Y, Luo B Analysis of pulmonary heme oxygenase-1 and nitric oxide synthase alterations in experimental hepatopulmonary syndrome. Gastroenterology. 2003; 125(5): 1441-51.

18. Ling Y, Zhang J, Luo B. The role of endothelin-1 and the endothelin $B$ receptor in the pathogenesis of hepatopulmonary syndrome in the rat. Hepatology 2004; (39): 1593-602.

19. Rabiller A, Nunes H, Lebrec D et al Prevention of gramnegative translocation reduces the severity of hepatopulmonary syndrome. Am J Respir Crit Care Med. 2002; (166): 514-17.

20. Thenappan T; Goel A; Marsboom G; Fang YH; Toth PT; Zhang HJ; et al. A central role for CD68(+) macrophages in hepatopulmonary syndrome. Reversal by macrophage depletion. American Journal of Respiratory and Critical Care Medicine. 2011 Apr; 183 (8):1080-91.

21. Zhang ZJ; Yang CQ. Progress in investigating the pathogenesis of hepatopulmonary syndrome. Hepatobiliary \& Pancreatic Diseases International. 2010 Aug; 9 (4):355-60.

22. Roberts KE; Kawut SM; Krowka MJ; Brown RS Jr; Trotter JF; Shah V; et al. Genetic risk factors for hepatopulmonary syndrome in patients with advanced liver disease. Gastroenterology 2010 Jul; 139 (1): 130-9.e24.

23. Chih-Hsin Lee MD, Shih-Tsung Cheng MD. Shortness of breath while sitting up: hepatopulmonary syndrome CMAJ, 2011 January; (183):1.

24. Peter Deibert, Hans-Peter Allgaier, Stefanie Loesch, Claudia Müller, Manfred Olschewski, Hinrich Hamm, et al. Hepatopulmonary syndrome in patients with chronic liver disease, role of pulse oximetry BMC Gastroenterology. 2006; (6):15. 
25. Amir Houshang Mohammad Alizadeh, Seyed Reza Fatemi, Vahid Mirzaee, Manoochehr Khoshbaten, Bahman Talebipour et al, Clinical features of hepatopulmonary syndrome in cirrhotic patients. World J Gastroenterol. 2006 March; (12): 1954-1956.

26. Vincent Ho. Current concepts in the management of hepatopulmonary syndrome. Vascular Health and Risk Management. 2008; 4(5) 1035-1041.

27. H. Page McAdams, Jeremy Erasmus, Robert Crockett, John Mitchell, J. David Godwin, Vincent G. McDermott. The Hepatopulmonary Syndrome: Radiologic Findings in 10 Patient sAJR 1996;(166):1379-1385.

28. Rajwal S. R, Davison S. M, R. Prasad, K. Brownlee and P. McClean. Successful liver retransplantation for recurrent hepatopulmonary syndrome Pediatr Transplantation. 2007; (11) 927-930.

29. El Makarem MA; Elakad A; Ali A; Abd-Elkader M; Sayed AF; Taha A; Et al. Hepatopulmonary syndrome, prevalence and predictors in Egyptian cirrhotic patients. Tropical Gastroenterology: Official Journal Of The Digestive Diseases Foundation 2011 Jan-Mar; Vol. 32 (1): 25-30.

30. Gupta S; Faughnan ME; Lilly L; Hutchison S; Fowler R; Bayoumi AM. Norfloxacin therapy for hepatopulmonary syndrome: a pilot randomized controlled trial. Clinical Gastroenterology and Hepatology: The Official Clinical Practice Journal of The American Gastroenterological Association. 2010 Dec; Vol. 8 (12):1095-8.

31. Sztrymf B, Rabiller A, Nunes H. Prevention of hepatopulmonary syndrome and hyperdynamic state by pentoxifylline in cirrhotic rats. Eur Respir J. 2004; (23): 752-8.

32. Zhang J, Luo B, Tang L, Wang Y. Pulmonary angiogenesis in a rat model of hepatopulmonary syndrome. Gastroenterology. 2009; (136): 1070-80.

33. Junlan Zhang, Bao Luo, Liping Tang, Yong Ming Wang, Cecil R. Stockerd et al. Pulmonary Angiogenesis in a Rat Model of Hepatopulmonary Syndrome Gastroenterology. 2009 March; 136(3): 1070-1080.
34. Rajasekhar Tanikella, George M. Philips, Dorothy K. Faulk, Steven M. Kawut, Michael B. Fallon. Pilot Study of Pentoxifylline in Hepatopulmonary Syndrome. Liver Transpl. 2008 August; 14(8): 1199-1203.

35. Binay K De, MD, Deep Dutta, MD, Subrata K Pal, MD, Subhabrata Gangopadhyay, MBBS, Sumanta Das Baksi, MBBS, and Adyapad Pani, MBBS. The role of garlic in hepatopulmonary syndrome: A randomized controlled trial Can J Gastroenterol. 2010 March; 24(3): 183-188.

36. Masud H. Khandaker, MD, PHD; Bettina M. Knoll, MD, PHD; Amindra S. Arora, Mbbchir. 63-YearOld Man With Cryptogenic Cirrhosis and Dyspnea Mayo Clin Proc. May 2008; 83(5):580-583.

37. Hemprich U; Papadakos PJ; Lachmann B. Respiratory failure and hypoxemia in the cirrhotic patient including hepatopulmonary syndrome. Current Opinion in Anaesthesiology. 2010 Apr; 23 (2): 133-8.

38. Chen K; Li B. Reversal of severe hepatopulmonary syndrome in chronic hepatic cirrhosis by living donor liver transplantation: report of two cases. Surgery Today. 2011 Mar; 41 (3):441-3.

39. Kremers K Walter, Marrije van IJperen, W. Ray Kim, Richard B. Freeman, Ann M. Harper, Patrick S. Kamath et al. MELD Score as a Predictor of Pretransplant and Posttransplant Survival in OPTN/UNOS Status 1 Patients. Hepatology. 2004; 39(3): 764-769.

40. Gupta S, H. Castelde, R. V. Raoa, M. Picarde, L. Lillyg, M. E. Faughnanaand, et al. Improved Survival After Liver Transplantation in Patients with Hepatopulmonary Syndrome American Journal of Transplantation. 2010; (10): 354-363.

41. Suliemana B. M, Hunsickerb L. G, D. A. Katzc and M. D. Voigta, OPTN Policy Regarding Prioritization of Patients with Hepatopulmonary Syndrome: Does It Provide Equitable Organ Allocation. American Journal of Transplantation 2008; (8): 954-964.

42. O'Grady J. G. and J. Wendon Pushing the Boundaries in Hepatopulmonary Syndrome.

Revista electrónica publicada por el Departamento de Farmacología de la Escuela de Medicina de la Universidad de Costa Rica, 2060 San José, Costa Rica. ${ }^{\circledR}$ All rights reserved. Licensed under a Creative Commons Unported License. 
American Journal of Transplantation 2010; (10): 203-204.

43. Hae Won Lee, MD, Kyung-Suk Suh, MD, Joohyun Kim, MD, Woo Young Shin, MD, Nam-Joon Yi, MD, Hwan Jun Jae, MD, et al. Pulmonary Artery Embolotherapy in a Patient with Type I Hepatopulmonary Syndrome after Liver Transplantation. Korean J Radiol. 2010 Jul-Aug; 11(4): 485-489.

44. Kochar R; Tanikella R; Fallon MB Serial pulse oximetry in hepatopulmonary syndrome. Digestive Diseases And Sciences. 2011 Jun; 56 (6): 1862-8.

45. Kazuko Y. Fukushima, Hiroshi Yatsuhashi, Akitoshi Kinoshita, Toshihito Ueki, Takehiro Matsumoto, Mitsuhiko Osumi, et al. Two cases of hepatopulmonary syndrome with improved liver function following long-term oxygen therapy J Gastroenterol 2007; (42):176-180.

46. Chihara Y; Egawa H; Tsuboi T; Oga T; Handa T; Yamamoto $\mathrm{K}$; et al. Immediate noninvasive ventilation may improve mortality in patients with hepatopulmonary syndrome after liver transplantation. Liver Transplantation: Official Publication of The American Association For The Study Of Liver Diseases And The International Liver Transplantation Society. 2011 Feb; 17 (2):144-8.

47. De Santis M; Vaz Rodrígues L; Matos F. Hepatopulmonary syndrome: case report and literature review. Revista Portuguesa de Pneumologia 2010 jul-Aug;16(4):679-85.

\section{INFORMACION DE AUTOR:}

Álvarez Arguedas, Karina

Email: kari_a28@yahoo.com 